\title{
Practice, Politics, and Papers
}

\author{
Eelco F. M. Wijdicks
}

Published online: 6 January 2009

(c) Humana Press Inc. 2009

At the beginning of a new year, it is good to again reflect on our journal. The journal, Neurocritical Care, has done well, received a respectable impact factor, and continues to publish interesting papers that impact on our daily practice. The journal is a society membership benefit and a good bargain. Moreover, the journal is part of the HINARI program (www.who.int/hinari) and is provided for free or at very low costs to developing countries (mainly in Africa, Eastern Europe, Central Asia, Australasia, Far East, Indian subcontinent, Central and South America).

Our 2008 statistics look good. The average number of days between the date the manuscript was received and the first editorial decision is 21 days. If we like what you send us we move quickly, do not bother with minutiae and try to get it published soon. The average time between acceptance and Online First publication has been halved and is now less than 3 weeks. Access to the journal is reliable and more readers and authors are showing interest. There was an 8-fold increase in the number of downloads compared with last year.

Our aim has remained fairly straightforward. Although we publish experimental studies and reviews on evolving technology, the journal, I believe, should remain a journal that regularly publishes on practice and politics.

Practice in the Neurosciences Intensive Care Unit may involve management protocols, changes in day-to-day practice and therefore manuscripts should have general applicability. We like to show how patients are managed, how their complications are handled, and how indications

E. F. M. Wijdicks ( $($ )

Division of Critical Care Neurology, Mayo Clinic, 200 First Street SW, Rochester, MN 55905, USA

e-mail: wijde@mayo.edu for certain procedures are defined. We are interested how trials are run and also in the results. Feasibility studies may not easily find a home, but Neurocritical Care will seriously consider these manuscripts. We may practice in an evidence free world but we like to change that.

Neurocritical Care is also interested in politics as we further define our field. Some of us appear they are on a providential mission, thrive with pushing the envelope and try to open a discussion on who does what and why. Others find progress in compromise in order to escalate the role in care. Neurocritical Care should welcome papers that address turf but we will not publish studies that are selective in the extreme. In forthcoming issues, important procedures such as percutaneous tracheostomy and ventriculostomy placement by neurointensivists will be discussed. These papers may create a sense of annoy, but other positions will be presented to create a counter punch. It is for the readers to decide which side to take and I believe these discussions can only be productive and helpful.

This year, we have the opportunity to post videos. This creates a new complexity (e.g., patient consent), but videotaping certain neurological features or neuro endovascular procedures or procedures at the bedside can be posted on the Springer site. We hope we will be able to post clips this year.

Every year we switch covers and I am again grateful for David Factor to provide such magnificent illustrations. The covers represent some aspects of neurocritical care and this year it is the brain-heart linkage. With every issue, we will change the colors so that the individual issues are better distinguished.

After five years, the editorial board is ready for some changes, and we will add the following editorial board members. We welcome Drs. Richard Bernstein, Anish 
Bjardwaj, Ricardo Carhuapoma, Jan Claassen, Jennifer Frontera, Romergryko Geocadin, Andreas Kramer, Giuseppe Lanzino, Peter Papadakos, Jose Suarez, and Bradley White. They all bring expertise in specific areas. Dr. Rabinstein will continue as a section editor with the main responsibility to write the section "Spanning the Globe". We thank Drs. Hacke, Hanley, Henderson, Guijar, Lancomis,

M. Giulia Abate

Imoigele Aisiku

Venkatesh Aiyagari

Paul Akins

Neeraj Badjatia

Hee-Joon Bae

Andrew Baker

Helen Barkan

Walter Bartynski

Mustafa Baskaya

Michael Bell

Randy Bell

Giorgio Berlot

James Bernat

Richard Bernstein

Eric Bershad

Anish Bhardwaj

Kathleen Bledsoe

Eric Bloomfield

Cecil Borel

Eric Bourekas

Robert Bryan

Joseph Burns

Norbert Campeau

Raphael Carandang

Ricardo Carhuapoma

Emmanuel Carrera

Arturo Chieregato

Charmaine Childs

Michael Christensen

Jan Claassen

Harry Cloft

Jonathan Coles

Jesse Corry

William Couldwell

Salvador Cruz-Flores

Marek Czosnyka

Viera Danielisova

Vincent Degos

Bart Demaerschalk

Rajat Dhar

Victor Diaz

Jens Dreir

Jonathan Edlow
Shorvon, and Vinuella. They have all been very supportive of the journal in its early years, and I thank them for their service. I also thank the Ad Hoc reviewers-listed below-who have been willing to review papers and improve on the quality of the manuscripts, and I am pleased to see the number of devoted reviewers is increasing. I am grateful for the time invested by them.

Tulin Erdem

Muhammad Farooq

Jeremy Fields

Matthew Flaherty

Alexander Flint

Isabel Fragata

Ognjen Gajic

Charly Gaul

Mark George

Dimitrios Georgiadis

Elias Giraldo

Timothy Girard

David Greer

Joshua Goldstein

J. Goncalves de Olivera

John Halperin

Moon Ku Han

Jacob Hansen-Schwartz

Tomoki Hashimoto

Michael Hehir

Sean Henderson

Hans Henkes

Ji Hoe Heo

Michael Hill

Dale Hoekema

Robert Hoesch

Roman Huber

J. Stephen Huff

Ferdinand Hui

April Hurdle

Olivia Hurtado

H. B. Huttner

Catalina Ionita

Max Jagersberg

Michael James

Tarig Janjua

Adrian Jarquin-Valdivia

Clay Johnston

S. Andrew Josephson

David Kallmes

Tadashi Kaneko

Thomas Kapapa

Vasilios Katsaridis

Emanuela Keller 
Salah Keyrouz

Naveed Khan

Matthias Klein

Matthew Koenig

Andreas Kramer

Erwin Kompanje

Pater Lackner

Joseph LaManna

Maarten Lansberg

Guiseppe Lanzino

Fin Stolze Larsen

Daniel Laskowitz

Christos Lazaridis

Kiwon Lee

Vivien Lee

Stephane Legriel

Denise Lemke

Peter LeRoux

Thomas Lescot

Joshua Levine

David Liebeskind

Geoffrey Ling

Juan Llompart-Pou

Joel MacDonald

R. Loch Macdonald

Adel Malek

Geoffrey Manley

Edward Manno

Niklas Marlund

Luciana Mascia

Anna Teresa Mazzeo

Robert McDonald

Nancy McLaughlin

Paul Michel Mertex

Chad Miller

Michael Moussouttas

Susanne Muehlschlegel

Masao Nagayama

Simon Nagel

Andrew Naidech

T. Jayashree Narayanan

Joseph Nates

Barnett Nathan

Neeraj Naval

Ivan $\mathrm{Ng}$

Thanh Nguyen

Marc Nuwer

Paul Nyquist

Art Pancioli

Ravi Patel

Konstantinos Paterakis

Harry Petrushkin

Axel Petzold
Hans-Walter Pfister

Thanh Phan

O. Piazza

Javier Provencio

Corina Puppo

Louis Puybasset

Gail Pyne-Geithman

Denise Rhoney

Carlos Romero

Jeffrey Rosenfeld

Guy Rordorf

Jonathan Rosand

Andrea Rossetti

Jean de Oliveria

Owen Samuels

Clemens Schirmer

Peter Schellinger

David Schiff

Stefan Schwarz

Woo-Keun Seo

Carrie Sims

John Sims

Huaxin Sheng

Lori Shutter

Martin Smith

Erik St. Louis

Latha Stead

Sherman Stein

Luzius Steiner

Thorsten Steiner

Jose Suarez

Marek Sykora

Jerzy Szaflarski

Robert Taylor

Shelly Timmons

Samuel Tisherman

Daoming Tong

Michel Torbey

Stella Tsirka

George Tsolakidis

Diederik van de Beek

Walter van den Bergh

Marjorie Wang

Nicolas Weiss

H. Christian Weston-Schumacher

J. Bradley White

Thomas Wolfe

Marla Wolfson

Wendy Wright

John Zhang

Douglas Zochodne

Alexander Zubkov

David Zygun 